\title{
Acceptance in Top Journals Shows Large Disparities across Name-inferred Ethnicities
}

Hao Peng ${ }^{1}$, Karim R. Lakhani ${ }^{2,3,4}$, Misha Teplitskiy ${ }^{1,3, *}$

${ }^{1}$ School of Information, University of Michigan, Ann Arbor, MI, USA

${ }^{2}$ Harvard Business School, Harvard University, Boston, MA, USA

${ }^{3}$ Laboratory for Innovation Science at Harvard, Harvard University, Boston, MA, USA

${ }^{4}$ Institute for Quantitative Social Science, Harvard University, Boston, MA, USA

Publishing in top journals is crucial to academic careers, but many fields show substantial ethnic disparities in publication counts, which may be caused by disparities in producing manuscripts or getting them accepted. Here, we investigate the latter mechanism using the peer review files of 16.5K manuscripts submitted between 2013-2018 to a field-leading biology journal (Journal A) and a middle-tier journal of similar scope (Journal B). The editorial data are supplemented with authors' name-inferred ethnicities and extensive controls including submissions' topic, author prestige, and citation impact, even for rejected-and-publishedelsewhere submissions. We find substantial disparities in acceptance across inferred ethnicities and that these are driven by the editors and not peer reviewers. In particular, for a given amount of future impact and other submission characteristics, papers by East Asian-named authors were 4.3-14.6 percentage points less likely to be accepted than those by British-originnamed authors. Journal $A$ editors were about 7.1-8.1 percentage points less likely to send East Asian-authored (Chinese and non-Chinese) papers out for peer review and, for a given level of reviewer enthusiasm, 7.2 percentage points less likely to ultimately accept them (nonChinese only). In contrast, peer reviewers gave recommendations that were similar across all name-inferred ethnicities. As science continues to globalize, these findings signal the need to better understand ethnic disparities in the review process.

\footnotetext{
* Correspondence may be addressed to Misha Teplitskiy, tepl@umich.edu
} 
Research consistently finds that individuals from minority social groups are underrepresented throughout the scientific career pipeline ${ }^{1+5}$. The widely used phrase "publish or perish" vividly summarizes that a key, if not most important, scientific activity contributing to persistence in the pipeline is publishing papers, especially in leading academic journals ${ }^{6}$. Understanding disparities in publishing is thus important for understanding broader social inequalities in science.

Across fields, different ethnic groups publish at different rates 778 . These disparities in publication counts may be related to disparities in the production of manuscripts or getting them accepted, but distinguishing between these mechanisms using publicly available data is difficult. Yet understanding the mechanisms separately is important, since they entail different institutions and actors and, consequently, different policy interventions.

Here, we focus on the latter mechanism, manuscript review. Disparities across social groups in review may arise through a variety of mechanisms. Scientific publishing in established journals typically involves several individuals, specifically editors and peer reviewers. The fundamental premise, or aspiration, of this system is that these individuals asses research based on its intellectual merit, regardless of authors' social identities 910 . If journals' evaluation processes live up to this aspiration, then disparities in publishing outcomes may be the result of disparities in merit. Disparities in submissions' merit may be the result of biases upstream in the research process, such as in obtaining funding, mentorship ${ }^{11}$, and other resources ${ }^{12 \mid 13}$, but these upstream biases need not imply biases on the part of editors and reviewers.

Disparities in publishing may also arise through non-merit mechanisms. First, evaluators may be directly biased, unconsciously or not, against particular social groups. Second, evaluators may rely on stereotypes. Evaluating the merit of complex ideas is difficult, and has arguably become only more so over time ${ }^{\sqrt{14}}$. The expertise needed to create and evaluate scientific papers is increasingly beyond the grasp of any specific individual ${ }^{[5 \mid 16}$. Consequently, decision-makers may try to facilitate evaluations by substituting costly-to-acquire but direct knowledge of manuscript content with "cheap" inferences made via stereotypes ${ }^{17-19}$, such as inferring from authors' names 
and locations their expertise, resource availability, or other factors related directly or indirectly to manuscript quality ${ }^{20}$. In principle, such inferences may be valid on average. For example, recent research finds associations between innovators' demographic characteristics and the types of innovation they pursue $21-23$. If the inferences are valid, they may not result in acceptance disparities across demographic groups, but may lock-in existing inequalities ${ }^{24}$, normalize discrimination ${ }^{25}$, and penalize (or reward) individuals who do not fit their group's average tendency.

However, stereotypes may be faulty. For example, they may reflect the past but fail to properly reflect the present in a quickly changing environment. In this case, disparities in acceptance conditional on merit may appear across demographic groups. In practice, evaluations using faulty stereotypes can create double standards across groups ${ }^{26}$. Specifically, if a disfavored group of submitting authors faces lower expectations of quality, their actual quality will need to be unusually high to overcome the expectations. Double standards may thus manifest empirically in lower acceptance rates for disfavored groups conditional on merit, but higher merit conditional on $\operatorname{acceptance}^{27}$.

Additionally, editors may also use criteria loosely related to merit, for example, discriminating on research topics in order to achieve a particular portfolio of topics. If demographic characteristics are associated with topics, disparities may appear without bias per se.

This discussion suggests that disparities in acceptance may or may not indicate bias on the part of editors and reviewers. It is thus important to consider a variety of mechanisms when interpreting disparities.

There is a lack of systematic evidence on ethnic disparities in review and the mechanisms driving them. Much of the related research investigates disparities related to gender, location, social connections, and prestige $10,27,-34$. Some studies suggest that women and underrepresented minorities face discrimination in perceptions of quality, obtaining research grants, and recognition for novel contributions $1220,22,35,36$. However, these findings of discrimination are far from unequiv- 
ocal, and some recent audit experiments find little evidence of gender or race discrimination 3738 .

The applicability of extant research to ethnic disparities in publishing has three limitations. First, many existing studies examine relatively quick and low-stakes evaluations, such as in responding to emails. In contrast, publishing involves time-consuming evaluations conducted by experienced and well-incentivized evaluators. Second, existing research typically focus on science in the U.S. and other Western countries. Yet scientific research is increasingly global ${ }^{39}$, with China now producing the most papers of any nation $\frac{40}{}$, while the reviewers and editors of major journals, are often based in the West ${ }^{41}$. It is thus important to study publishing inequalities among ethnic groups defined from a global perspective. Third, many existing studies focus on the early part of research projects-obtaining funding. Evaluating applicants is generally a formal part of funding competitions $^{\sqrt{36}}$, so it is possible, and maybe even likely, that applicants' ethnic or other social identities affect evaluations. In contrast, authors' identities should in principle play no role once the research is complete and submitted for publication. To our knowledge, there have been no studies of global ethnic disparities in publishing in top journals. This is the gap this study aims to fill.

We partnered with the editors of two major journals in biology (see Data and Methods for details), which we call Journal A and Journal B to ensure anonymity, and obtained from them the review files of all submissions between 2013-2018. Journal A is field-leading and career-making while Journal B is middle-tier. Both journals use the single-blind review - authors' identities are visible to editors and reviewers. We measured authors' perceived ethnicity and gender algorithmically, using their first and last names with the classifier Ethnea ${ }^{42}$ Data and Methods. We call this name-inferred ethnicity "perceived" to distinguish it from authors' self-identifications, which are at present rarely collected by publishers ${ }^{43}$. We adopt the term "ethnicity" instead of "nationality" because decision-makers in our data can directly observe the national location of authors' institutions, so "ethnicity" reflects a richer set of characteristics decision-makers can infer from names above and beyond location. Using ethnicities determined via algorithm has several important limitations (discussed in Data and Methods). However, lacking detailed data on authors' self-identities, reviewers and editors may make inferences similar to those of the algorithm. Over- 
all, the findings should be interpreted as only suggestive of associations between publishing and ethnic self-identities, a point we return to in the discussion.

Although most submissions were authored by several individuals, we assigned the perceived ethnicity and gender to each manuscript based on the name of its last author, given the tendency in biology for the last author to be seen as inspiring, funding, and managing the research 12 444 45 . We placed authors into 9 broad ethnic categories based on geographic regions and the number of observations (Data and Methods and SI Table 1).

To better understand the mechanisms producing possible disparities, we sought to capture additional information about each submission, particularly its quality. We used the Microsoft Academic Graph (MAG) bibliometric database to supplement the editorial data with key covariates, including author prestige, manuscript topics, and, crucially, manuscript novelty and citation impact (Data and Methods). We measured the novelty of manuscripts algorithmically, using atypical combinations of references ${ }^{46}$. We measured two aspects of citation impact: impact amount is quantified as citations counts and impact disruptiveness is quantified by how much a paper replaces its predecessors in the eyes of future citers ${ }^{47}$. We refer to these measures of novelty and citation impact as proxies for manuscript "quality."

\section{Disparities in Publishing Success}

Submissions over time. The submission volume across perceived ethnicities changed over time. We focused on Journal $A$ for this analysis due to its more complete submissions data relative to Journal B. Among all submissions to Journal A, 27.3\% were from British-origin authors; $20.9 \%$ were Western \& Northern European; 20.7\% were Chinese; 9.4\% were Southern European; 8.3\% were non-Chinese East Asian; the remaining 13.1\% were from Middle Eastern, Indian, Eastern European, and African authors. 0.4\% submissions were labeled as "Unknown Ethnicity". The relatively small number of observations for African authors $(\mathrm{N}<50)$ prevented us from reaching robust conclusions, and these observations were excluded in the subsequent analysis. 


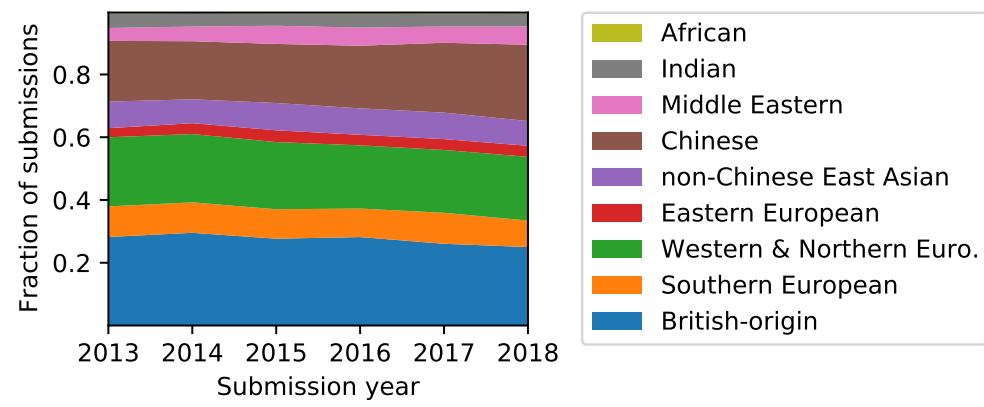

Figure 1: Statistics of Journal A submission data. The percentage of submissions from each ethnicity between 2013 and 2018. Note that African-named percentages are invisible due to a relatively small number of submissions.

Fig. 1 shows how the perceived-ethnicity composition of submitting authors changed over time. Submissions from Chinese authors, the third largest group, increased by about half from 2013 to 2018. By the year 2018, the number of Chinese submissions was comparable to British-origin submissions.

Disparities in acceptance. Turning to disparities in acceptance, we examined the association between author ethnicity and acceptance rates through a series of increasingly parameterized logistic regression models, with an indicator variable ( $1=$ Accepted, $0=$ Rejected) as the outcome (see SI Tables S2-S3 for model estimates). For each model, we calculated the average marginal effects (AMEs) of perceived ethnicities on the probability of being accepted, with British-origin names as the reference category. The AME for a particular ethnicity reflects the difference (averaged over all observations) in the acceptance probability compared to British-origin names, holding other variables constant. To aid exposition, we refer to all non-British-origin ethnic groups as "minority," even though numerically many of these groups are well represented among submissions. The following results should be interpreted as associations rather than causal effects.

Focusing first on Journal A, Model 1 reflects raw differences in success rates by last author perceived gender and ethnicity. The rates reveal that submissions from all minority ethnicity authors were significantly less likely to be accepted compared to British-origin authors. The 

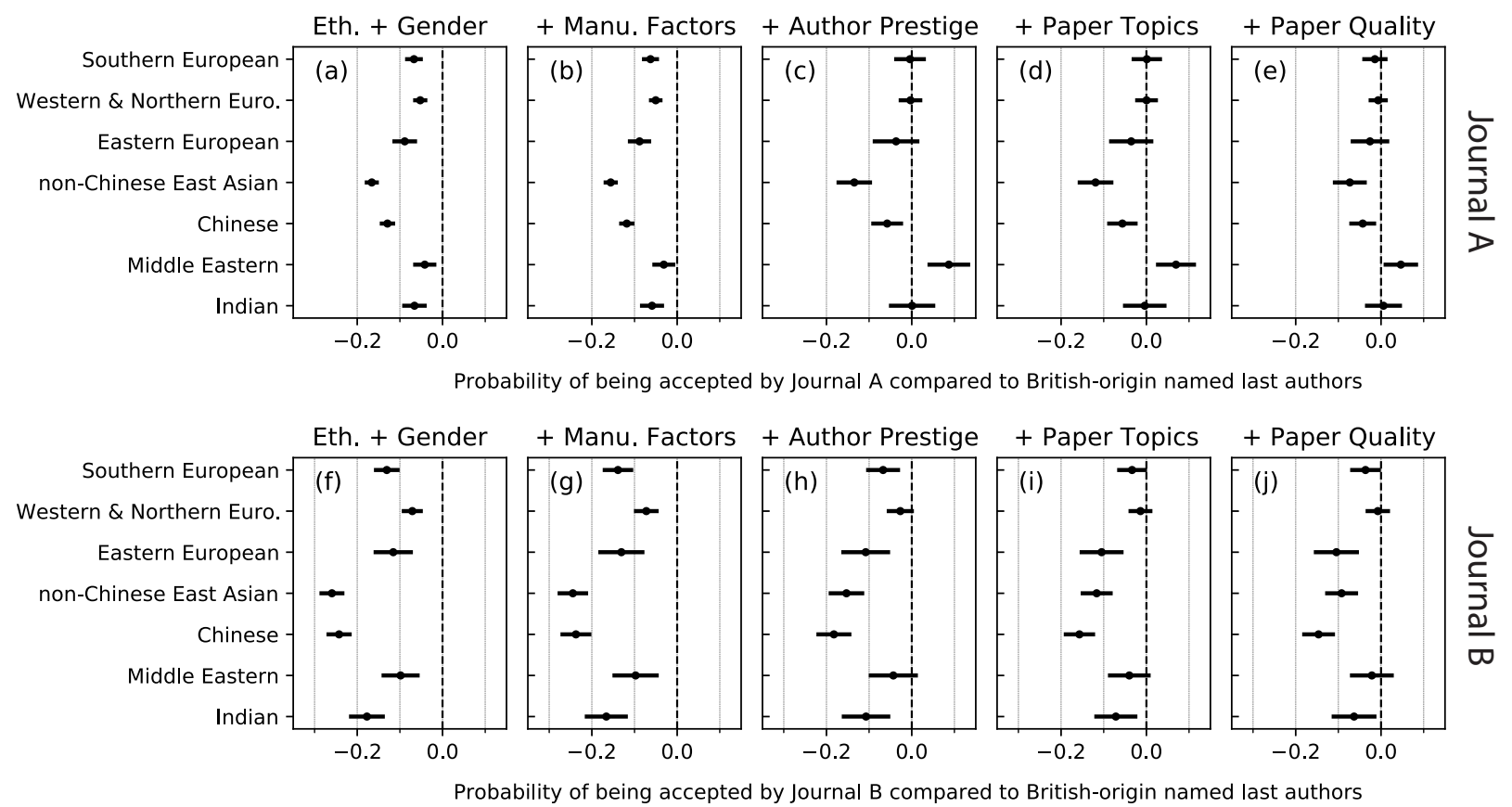

Figure 2: The average marginal effects of author ethnicity on the final acceptance rate. ae, For submissions at Journal A. a, Model 1 includes only author perceived ethnicity and gender (16,956 observations). b, Model 2 adds manuscript factors (16,954 observations). c, Model 3 adds author prestige (7,062 observations). d, Model 4 adds controls for paper topics (7,062 observations). e, Model 5 adds paper quality and impact including novelty, disruption, and log citations (6,947 observations). f-j, For Journal B, there are 14269, 8874, 7195, 7195, and 6365 observations in Models 1-5, respectively. Error bars indicate 95\% bootstrapped confidence intervals.

magnitude of the disparities (Fig. 2a) varies across groups: European- and Asian-named authors experience 5 and 15 percentage point lower acceptance, respectively. Model 2 shows that the magnitude of disparities is substantively similar after controlling for manuscript factors including submission year, the number of authors, title and abstract length, and abstract readability (Data and Methods). While all these factors are associated with acceptance (SI Tables S2-S3), they had limited influence on the magnitude of ethnic disparities. For example, compared to Model 1, there is only 1 percentage point drop in the acceptance gap for three Asian ethnicities (Fig. 2b).

Model 3 adds factors related to author prestige, including last author's rank, number of pre- 
vious publications, and affiliation rank and location (U.S.-based or International; see Data and Methods). Adding author prestige factors makes the acceptance rates for European and Indian authors no longer significantly different from British-origin authors. The disparities for the two East Asian groups decrease by about 4.5 percentage points (Fig. 22). The acceptance probability for Middle Eastern-named authors was even higher than that for British-origin after controlling for prestige. In other words, at similar prestige levels, Middle Eastern authors were accepted even more often than British-origin authors.

Adding controls for paper topics in Model 4, we find that the marginal effects change little, although the disparity for non-Chinese East Asian authors drops an additional 1.6 percentage points (Fig. 2d). This suggests that the topics that authors work on either did not vary systematically by ethnicity or did not vary in how much they were favored by editors and reviewers.

Finally, Model 5 controls for paper novelty and citation impact. The disparities for East Asian authors further decrease by 1.3-4.7 percentage points. Nevertheless, even in this stringently controlled model, compared to British-origin-named authors, the acceptance rates for the two East Asian-named authors were 4.3 and 7.3 percentage points lower (Fig. 2e).

In summary, observable characteristics of the submissions account for many, but not all, of the disparities across perceived ethnicities. For European and Indian authors, disparities are not statistically different from 0 when accounting for factors related to author and affiliation prestige. Middle Eastern authors' disparity is accounted for by the combination of author prestige and paper quality. However, while accounting for these characteristics substantially decreases the magnitude of acceptance disparities for East Asian authors, meaningful disparities nevertheless remain.

Repeating this analysis for Journal B (Fig. $2 \mathrm{f}-\mathrm{j}$ ) shows even larger disparities for East Asiannamed authors compared to Journal A (4.3 vs. 14.6 for Chinese and 7.3 vs. 9.2 for non-Chinese East Asian). Additionally, the disparities for Indian- and Eastern European-named authors are now larger and statistically significant. These results raise the possibility that ethnic disparities 
increases as one moves down the journal status hierarchy, although we cannot rule out differences in unmeasured manuscript and reviewer characteristics. For example, it is possible that editors appear to "require" above-average reviewer enthusiasm for non-Chinese East Asian submissions because of a desire to achieve a particular portfolio of fine-grained topics, which our coarse-grained measures control for imperfectly.

We also controlled for author gender in the five regression models. Although our focus is ethnicity, we briefly discuss the association between gender and acceptance. Submissions from female authors had 1.6 percentage point lower acceptance rate compared to those from male authors based on Model 1. However, this gap disappears after controlling for manuscript factors such as the submission year, number of authors, and title and abstract characteristics (SI Tables S2-S3). The observed raw gender disparity is likely to be driven by non-East Asian ethnicities since their names usually do not clearly signal gender and are mostly classified as "Unknown" in our data.

The Role of Location. The full model (Model 5) controls for the location of last author's affiliation (U.S. or International), and shows that international submissions were significantly less likely than U.S. ones to be accepted (SI Tables S2-S3). This association corroborates other studies of peer review which find that manuscripts from relatively wealthy countries such as the United States are evaluated more favorably than those from poorer countries $\frac{3031}{48} 49$. The fact that disparities for East Asian-named authors appear despite controlling for location suggests that location is not the sufficient explanatory factor of the disparity.

However, location may still account for some of the magnitude of disparities. In particular, editors and reviewers of the two journals we study may be more familiar with U.S.-based authors and their affiliations. Greater direct knowledge of authors and affiliations may reduce reliance on indirect but easily-observable cues, such as names. Furthermore, submissions from U.S.-based and international authors may vary in the quality of their English writing, i.e. papers written by non-native English Speakers may be more difficult for editors and reviewers to assess. We expect differences in the quality of writing to be relatively small among U.S.-based submitters and, con- 
sequently, hypothesized that acceptance would be associated with perceived ethnicity less strongly among U.S.-based authors. To test this hypothesis, we fitted Model 5 separately to submissions from U.S.-based and international authors.

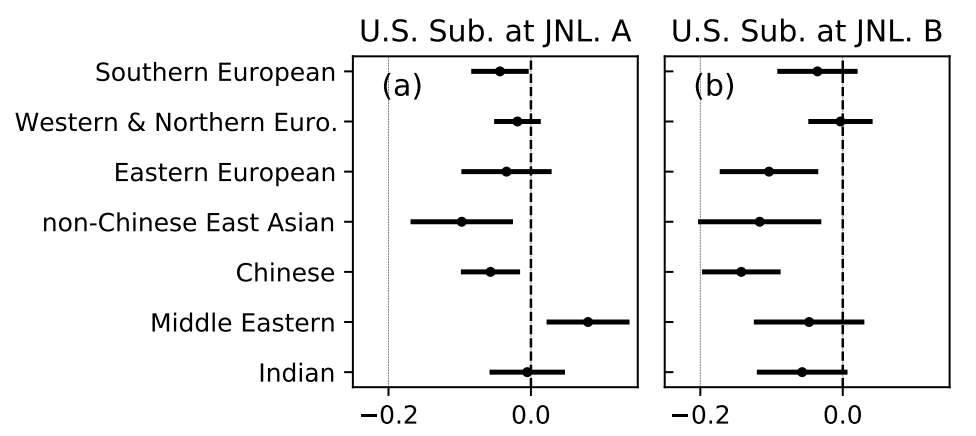

Prob. of being accepted compared to British-origin named last authors

Figure 3: The average marginal effects of ethnicity on the acceptance for U.S. submissions. The specifications of Model 5 was fitted to U.S. submissions for each journal. a, Based on 4,075 U.S. submissions at Journal A. b, Based on 2,812 U.S. submissions at Journal B. Note that these submissions were all published in the literature as Model 5 includes variables such as citations that are only available for published papers. Error bars indicate $95 \%$ bootstrapped confidence intervals.

The average marginal effects in Fig. 3 indicate that submissions from U.S.-based East Asian authors had 5.7-9.7 percentage points lower acceptance rates than their British-origin counterparts at Journal A, and about 11.7-14.2 percentage points lower at Journal B.

For Journal A, the disparities are significant for East Asian authors in the U.S. subset (SI Tables S4), and are negative but imprecisely estimated and not statistically significant in the international subset. However, the coefficients of East Asian disparities among international authors are not statistically different from that among the U.S-based subset, suggesting that international East Asian authors likely had lower acceptance rates than international British-origin named authors. This is also confirmed by including interactions between ethnicity and location in Model 5 in the full data, as no interaction terms have a statistically significant negative effect. We observed a more clear pattern at Journal B, as the perceived ethnicity coefficients for the U.S. subset and the international subset are both significantly different from 0 , but are not statistically different from 
each other for East Asian-named authors (SI Tables S5). Overall, the subgroup analyses show that the disparities are similar for U.S.-based and international submitters, suggesting that location is unlikely to explain much of the disparities across perceived ethnicity. Additionally, this pattern further supports the premise that names signal ethnicity and not simply location.

\section{Locating Disparities in the Evaluation Pipeline}
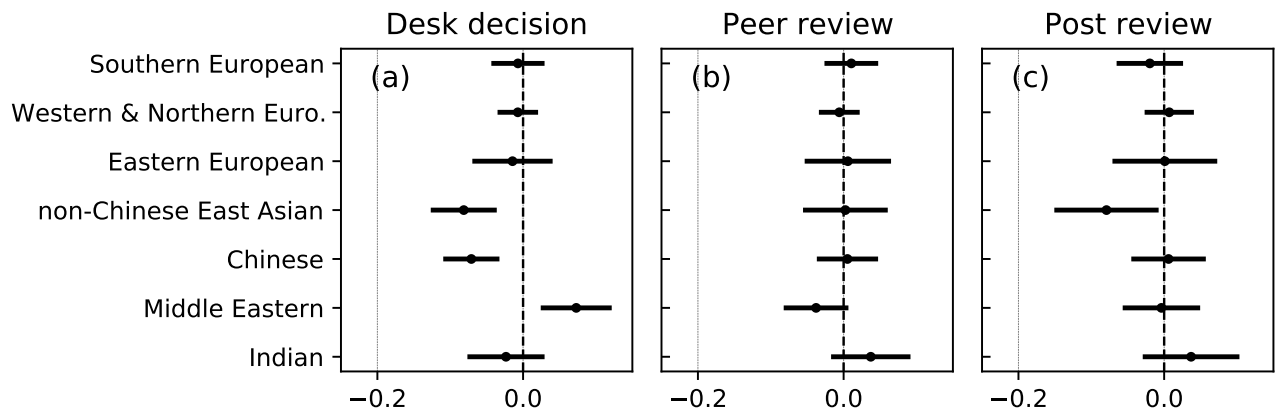

Prob. of receiving a positive decision at each stage compared to British-origin named last authors

Figure 4: The average marginal effects of ethnicity for three review stages at Journal A. The marginal estimations are based on a Model 5 estimated on ultimately-published submissions as it controls for paper citation impact. a, Each submission is an observation for the desk-decision stage. 3,331 out of 6,972 observations (47.8\%) were not desk-rejected. b, For the peer-review stage, we focused on the first round of review for each manuscript. Each (submission, reviewer) pair is an observation in the regression. 5,399 out of 9,077 observations (59.5\%) received a positive recommendation (Revision or Accept) at this stage. Reviewer random effects are included in the model. c, We used the first round of each submission as an observation in the post-review stage. 2,217 out of 3,294 observations (67.3\%) received a positive editorial decision (not being rejected) after the first round of peer review. The model also controls for the average reviewer recommendation score. Error bars indicate $95 \%$ bootstrapped confidence intervals.

Manuscript evaluation usually involves a number of steps and decision-makers, so it is not clear from the overall acceptance patterns where in the pipeline the disparities arise. Here, we take advantage of fine-grained data from each step of the evaluation pipeline at Journal A to better locate the source of the disparities. 
At both journals, manuscripts proceed through (1) desk decision and, if sent out for review, (2) external peer review and (3) post-peer review decision. Whereas the first and the third steps involve editors, the second step entails external peer reviewers. Each stage may contribute differently to ethnic disparities in the final acceptance rates. To examine the contributions, we relied on the more complete submission data from Journal $A$ and fitted a separate regression Model 5 using data from each stage. Since our full model controls for paper citation impact, which is only available for published papers, we focused on submissions that were eventually published somewhere and successfully located in the literature.

Desk-decision stage. Journal A desk-rejects a substantial fraction of the submissions. To model desk rejection as a function of last author perceived ethnicity and other submission characteristics, we fitted a logistic regression model with an indicator variable desk-rejected (coded as 0 ) or not (coded as 1) as the outcome.

Fig. 4a shows that manuscripts from East Asian-named authors (Chinese and non-Chinese) had 7.1-8.1 percentage points lower probability to be sent out for review than those from Britishorigin-named authors, keeping all other variables constant. The model reveals no statistically significant disparities for European and Indian authors. However, Middle Eastern-named authors were favored by 7.3 percentage points at this stage (Fig. 22).

Peer review stage. For the peer review stage, we considered only submissions that were sent out for external review. A manuscript can have several rounds of reviews. To ensure the independence of reviewer recommendations, we used only the first round of reviews for each submission, as reviewers are typically informed of other referees' comments after the first round. We defined the indicator variable "Accept or R\&R" as 1 if the reviewer gave a positive recommendation, including "Minor revision", "Major revision", and "Accept". Negative recommendations such as "Reject" were coded as 0 . To account for differences in reviewers' overall tendency to make positive recommendations, we included in Model 5 random effects for each reviewer (while our data do not include reviewers' identities, they include anonymized reviewer IDs). 
Fig. 4b shows that peer reviewers do not appear to have any systematic preference for or against authors of any perceived ethnicity.

Post-review stage. After each round of peer review, the editor selects a decision ("Accept", "Minor Revison", "Major Revision", or "Reject") based on reviewers' recommendations. We focused on the first round of review, due to decisions in subsequent rounds (where those exist) being likely highly correlated with the first decision. The dependent variable is the editorial decision for the first round, coded as 0 if it ended with a "Reject" decision and 1 if it led to another round of review or "Accept." We included in the regression a variable that we call "reviewer enthusiasm," defined as the reviewer recommendation ( 0 or 1 , defined above) averaged across all reviewers.

Fig. 45 shows that, similar to the desk-review stage, manuscripts from non-Chinese East Asian authors had lower acceptance (7.2 percentage points lower) in the post-review decisions, despite conditioning on reviewer enthusiasm, citation impact, and other observable characteristics. Given that the overall positive rate is $67.3 \%$ in the data sample at this stage, the observed disparity is relatively small, compared to the desk-decision stage (Fig. 4h). The disparities observed at this final or near-final stage indicate that editors exercised additional filtering after peer review, and this filtering resulted in the additional ethnic disparity for non-Chinese East Asian authors.

If these disparities reflect editors' bias, it is likely manifested in holding minority ethnicitynamed authors to a higher standard. The key empirical signature of such double standards is that the discriminated groups that are able to meet the higher bar are, on average, of higher quality than favored groups. To test for the double standards hypothesis, we again used the reviewer enthusiasm as a measure of paper quality. We compared the average reviewer enthusiasm for papers accepted at Journal A (using only the first round of review). As predicted, Fig. 5 shows that non-Chinese East Asian accepted papers have on average higher reviewer enthusiasm than Britishorigin accepted ones ( 0.83 vs. $0.74, \mathrm{p}=0.008$ ). Published Chinese-named papers have higher reviewer enthusiasm than British-origin ones (0.764 vs. 0.737, $\mathrm{p}=0.089$ ), but the difference is imprecisely estimated and not statistically significant at the conventional levels. 


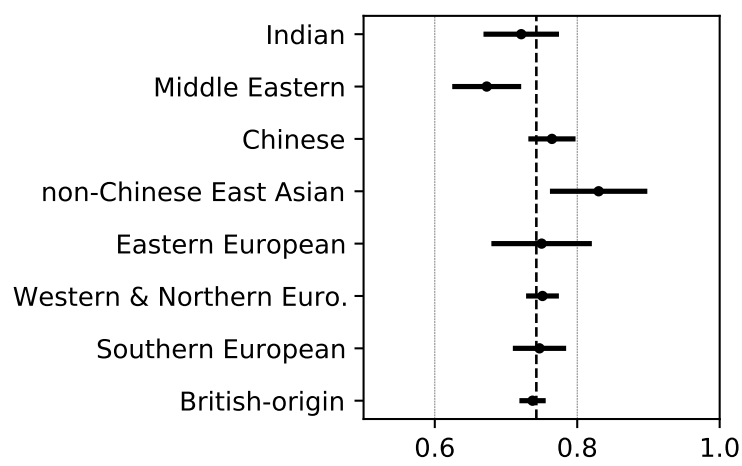

Avg. reviewer recommendation score

Figure 5: Mean reviewer recommendations for accepted paper at Journal $\boldsymbol{A}$. We used only the first round of review in the calculation. Reviewers' recommendations were quantified as 1=Accept or $\mathrm{R} / \mathrm{R}$, and $0=$ otherwise. The dashed vertical line is the mean score over all accepted papers. Non-Chinese East Asian papers have a statistically higher mean recommendation score than that of British-origin ones (0.83 vs. $0.74, \mathrm{p}=0.008)$. Error bars indicate $95 \%$ confidence intervals.

\section{Are citations a biased measure of impact across perceived ethnicities?}

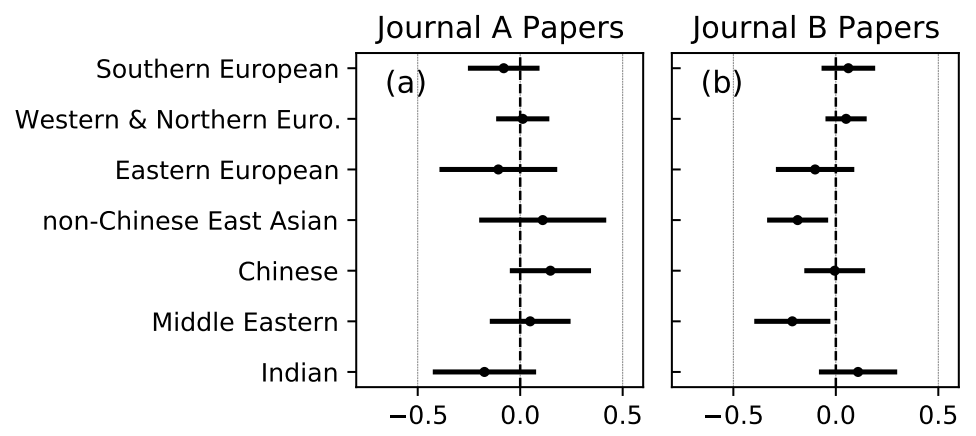

Increase in log citations compared to British-origin named last authors

Figure 6: The average marginal effects of ethnicity on the log number of citations. The specifications of Model 5 (excluding paper citations) was fitted to submissions for each journal. a, Based on 1,976 submissions accepted by Journal A (accepted manuscripts with missing values on any variables were excluded in the regression). b, Based on 3,398 submissions accepted by Journal B. The reviewer enthusiasm is included in the regression for only Journal A as we do not have the peer review data for Journal B. Error bars indicate $95 \%$ bootstrapped confidence intervals.

Our research design relies on being able to control for papers' underlying quality, which we 
do primarily using citations. However, existing research has argued that citation counts themselves may be biased against particular demographic groups ${ }^{50}$. If citations do not accrue similarly to different ethnic groups for similar contributions, then controlling for them may understate (or overstate) the true ethnic disparities in acceptance. To assess this possibility, we investigated whether authors of different ethnicities receive similar amounts of citations for papers of similar quality.

We assumed that papers published in the same journal with similar reviewer enthusiasm and other covariates are, on average, of similar quality. To see whether such papers receive similar citations across author ethnicities, we fitted a linear regression model with the log-citations as the outcome (raw citation counts produced similar results). We included all non-citation related covariates from Model 5, as well as "reviewer enthusiasm" (mean reviewer recommendation).

The average marginal effects in Fig. 67 show that papers published in Journal A by different ethnic groups received statistically indistinguishable numbers of citations conditional on covariates. This result suggests that, in this subset of papers accepted at Journal A, citations are not directly biased by perceived ethnicity. It is nevertheless possible that ethnicity and citations are related indirectly in all published papers: authors' ethnicity may affect which journal accepts a paper, and the journal may affect citations 51 . Specifically, if the field-leading Journal $A$ is less likely to accept papers from minority ethnicity-named groups, these papers will later receive fewer citations once published in lower-tier journals. In other words, citations would be downward-biased relative to the paper's true potential for impact. Yet the ethnic disparities in acceptance still appear despite controlling for the possibly downward-biased citation counts. In sum, if citations are ethnically biased, it is likely that the true disparities in acceptance at Journal A are even larger than those observed in Fig. 2e.

In contrast, Fig. 6b shows that among papers published in Journal B, relative to British-origin authors, non-Chinese East Asian and Middle Eastern authors received on average 0.19 and 0.21 $\log$ points fewer citations (12.3\% and $13.5 \%$ relative decreases). Because we lack the reviewer recommendation data for Journal B, it is unclear if the citation disparities would have been equalized 
with a control for reviewer enthusiasm. If these disparities did indeed occur for contributions of equal quality, then the acceptance disparity at Journal B for non-Chinese East Asian authors may be even higher than it appears in Fig. 2j, because the control for impact is itself biased.

Overall, we do not find a strong evidence of ethnic disparities in citations, and the slight disparities that appear at Journal B suggest that the disparities in acceptance are even larger in reality than shown in Fig. 2 .

\section{Discussion}

Disparities in publishing success across perceived ethnicities. Understanding disparities in publishing in top journals is crucial for understanding broader inequality in science, yet the evaluation processes leading to publication, particularly by the top journals, are very often opaque. Here, we used unusual access to the review files from a field-leading biology journal, as well as a mid-tier journal with similar scope and near-identical evaluation process, to examine disparities in acceptance by last author ethnicity, inferred from the author's name.

In raw terms, acceptance rates for all minority-ethnicity names are lower than that of Britishorigin ones, who have an acceptance rate that is nearly 20 percentage points higher than of the lowest-accepted groups (Fig. 2). These raw differences may be caused by a number of factors other than bias, such as paper quality and topic. Indeed, manuscript factors (submission year, number of authors, title and abstract length, and abstract readability), author prestige (such as author rank, affiliation rank and location, prior publications at two journals), paper topics, and paper novelty and citations all had strong associations with acceptance (SI Tables S2-S3).

Adding these various factors to increasingly parameterized regressions showed that disparities for European-, Indian-, and Middle Eastern-named authors are accounted for by author pres-

tige. Whether this pattern reflects a prestige bias ${ }^{1033}$ is unclear, because author prestige may be correlated with papers' merit or journal fit. 
However, all these measured factors, including paper topic, novelty, and impact, do not fully account for the lower acceptance rates of East Asian-named authors: net of those factors, their submissions had 6 and 12 percentage points lower acceptance rates than those from British-originnamed authors at Journal A and Journal B, respectively (Fig. 2). These disparities appear even among authors based in the U.S., making it unlikely that differences in English proficiency are the key driver of acceptance gaps. Indeed, the ethnic disparities among U.S.-based authors were of similar magnitude to that among international authors, especially at Journal B (Fig. 3 and SI Tables S4-S5). The practical significance of these disparities is very substantial. Across fields, top journals have acceptance rates as low as single digits. Consequently, a 6 percentage points acceptance reduction from an already small rate implies that publication is extremely improbable for all but a few submitters.

Mechanisms. The detailed data from Journal A enabled us to locate the source of these disparities in the evaluation pipeline. Disparities for East Asian-named authors occurred mainly at the desk-review and final review stages. Crucially, we find no systematic ethnic disparities in peer reviewers' recommendations. This finding is important for two reasons. First, it shifts focus to editors as the key decision-makers in understanding the disparities. Second, it makes less likely that the analysis is afflicted by measurement error and omitted variable bias. Specifically, a plausible explanation for our results is that an important confound differs across name-inferred ethnic groups, but is unmeasured or measured with error. Such errors, if present, should affect both editors' and reviewers' decisions. The fact that they do not suggests that the disparities arise in editors' decisions due to non-quality related factors or that editors assess different dimensions of quality.

Overall, the findings show that the large disparities in publishing in top journals, at least in biology, is not simply the result of disparities in the volume of manuscripts different ethnic groups produce. Instead, the review process, and particularly editors, are a major contributor to these disparities. The analysis does not, however, imply discrimination by editors of both journals, as the results are consistent with other mechanisms as well. One non-discriminatory mechanism is 
that editors at Journal $A$ assess manuscripts on different dimensions than peer reviewers, e.g. on topical fit to the journal, and these editor-valued characteristics may be associated with perceived ethnicities and not captured by our topical variables. Another candidate mechanism is that some ethnic groups submit their papers when they are less polished than the submissions of others, resulting in lower acceptance. However, since our analysis relies on the published versions of the papers, particularly through their citations, this difference in readiness may be unmeasured.

Limitations and directions for future research. Our study has a number of limitations, which we hope serve as fruitful directions for future research. First, our data do not include self-reported author ethnicity. This limitation is especially problematic for our analysis in cases where the author-identified ethnicity is known to evaluators and is different from that perceived from author names, i.e. as inferred by the algorithm or naive observer. Furthermore, we do not address multiethnic identities or key racial/ethnic groups, such as African American. Second, some ethnicities, such as Caribbean, Polynesian, and African had too few submissions to enable robust statistical comparison, and thus were excluded in the analysis and interpretation. Third, the research design we employed is not suitable for gold-standard causal claims. Consequently, we cannot rule out that the observed disparities were driven by some unmeasured differences between the submissions. We hope our research stimulates experimental studies that are better suited for establishing causality. Fourth, our analysis entailed a substantial amount of missing data. We focused on submissions that were eventually published in order to control for key confounding factors, such as papers' potential for impact. This analysis choice excluded a large fraction of submissions that could not be linked to their published versions. If these missing data is associated with ethnicity, then the measured ethnic disparities may be inaccurate. However, an important consideration for how the missing data may affect our results must contend with the fact that the editors' decisions showed statistically measurable disparities but the peer reviewers' recommendations did not, despite using the same imperfect data and measures. Furthermore, many controls such as paper topics and novelty scores were based on the published version of a manuscript, which might be different from its submitted version. Nonetheless, a related study using the same data shows that there is a high correlation between the submitted novelty and the published novelty 5 . Fifth, the analyses revealed 
that the ethnic disparities were larger at Journal B than at Journal A, despite similar topical scope and nearly identical reviewing procedures. This difference deserves further investigation, ideally across journals using very different review processes and in different domains, which would also aid in generalizability.

Lastly, research in decision-making consistently shows that biases can arise when cognitively challenging evaluation are hurried, resulting in evaluators leaning on heuristics 53,54 . If this mechanism was indeed operative, a natural intervention is to enable editors to evaluate slower. This mechanism may also explain why the disparities are observed at the desk reject stage (faster decision) but not the peer review stage (slower decision).

Despite these limitations, our study sheds light on the selection process at major journals and reveals substantial disparities across name-inferred ethnicities. These findings complement studies of publishing amount, showing that some differences in amount may be due not to lack of trying, but lack of success in those attempts. We hope these results can stimulate more finegrained analyses and, crucially, the collection of relevant data such as self-reported identities ${ }^{43}$. If supported by further research, the ethnic disparities could call for policy interventions. For instance, journals may switch to double-blind review or solicit additional opinions on manuscripts from rarely accepted ethnic groups.

\section{Data and Methods}

\section{Journal A submissions}

We use the full set of submissions to Journal A between 2013 and 2018. Each submission includes rich metadata, including all intermediate decisions and their dates. We removed from further anal-

ysis 72 submissions with missing first decision, 3 submissions with an organization as the last author, and 1 submission from a last author coded as Caribbean (Section 4) 
The data include the document object identifier (DOI) for manuscripts that were ultimately published in the literature and successfully located, including those published by Journal A and those published by other journals. We were able to locate 9,814 published papers (both accepted and rejected) based on DOI using the Microsoft Academic Graph (MAG) database (accessed in June 2019), which is the largest public bibliometric dataset ${ }^{55} 56$. We also obtained additional control variables for each paper using MAG, including author prestige, paper topics, and paper quality (Section 5).

The data include peer review reports and recommendations for manuscripts that were sent out for external review. A manuscript can be reviewed by several referees and over several rounds of review, although we focused on the first review rounds. The data included an anonymous Reviewer ID, which enabled us to control for the reviewers' overall tendencies to recommend acceptance or rejection in the cases in which reviewers reviewed multiple manuscripts.

\section{Journal B data}

The data for Journal B were less complete. It was missing peer review reports and the year of submission. For the latter, we use the year of publication as an approximation. However, the data identify submissions that were rejected between 2013 and 2018, published elsewhere, and successfully located (with DOI). We were able to retrieve 14,270 published papers (by Journal B or other journals) from MAG based on DOI, and obtained many control variables for these papers (Section 5). The final analytic sample consists of 14,269 observations, after excluding 1 paper from an author coded as Caribbean.

\section{Submitted vs. published versions}

Many of the key variables in our analysis come from MAG, which contains data on published rather than submitted papers. A limitation of using papers' published versions is that some of these covariates may change during the review process. However, existing work shows that the core 
content of manuscripts tends to change little from submission to publication, with most changes

occurring in framing and tone 57 61 . Furthermore, some important covariates, such as the number of authors or their prominence, is unlikely to change substantially from submission to publication.

\section{Ethnicity and gender coding}

Perceived vs. self-identified ethnicity. Relying on perceived (name-inferred) ethnicities comes with several limitations. First, perceived ethnic identities may differ from authors' self-identifies, which may be only moderately associated with geographic location. Second, authors may change last names, for example after marriage, further weakening the inferences based on current last names. Third, authors may identify as multi-ethnic, whereas we assign each author to just one group. Fourth, in some cases, reviewers and editors may have personal knowledge of the authors and know how they self-identify. Overall, these limitations of inferring ethnicity from names also likely apply to the perceptions editors and reviewers form, in most cases.

Classifier. We used Ethnea to infer author gender and ethnicity using author names ${ }^{42}$. Ethnea is trained using the PubMed database, with the location of the authors' affiliations as the ground truth. For a specific name, Ethnea assigns the ethnicity probabilities among matched authors. In the case of two or more predicted ethnicities, we took the one with the highest probability. It also provides ethnicity-specific gender predictions, such as Italian vs. English "Andrea." Ethnea has been shown to perform better than other machine learning approaches that rely on model training and feature selection of names 426263 .

Author names were cleaned of suffixes, such as $\mathrm{PhD}, \mathrm{MD}$, and their variations. We placed 26 individual ethnic classes defined by Ethnea into 11 high-level categories (SI Table S1). We dropped manuscripts from organizations and ethnicities with less than 5 observations (including Caribbean and Polynesian). The largest group, British-origin, is used as the reference category for ethnicity in the regression analysis. Ethnea offers binary gender predictions including Female and Male (used as the reference category in the regression). Names that are not recognized by Ethnea 
were assigned "Unknown" for both gender and ethnicity.

We categorized manuscripts into different perceived gender and ethnic groups based on their last authors. In case of solo-authored papers, we treated the single author as the last author.

\section{Control variables in regression models}

Many factors can influence manuscript evaluation, which could potentially confound the effect of ethnicity. We thus included many control variables. For Journal A, only the manuscript factors were based on the submitted manuscript; all other controls were based on its published version (including author prestige, paper topics and quality). For Journal B, the author names (therefor ethnicity and gender prediction) and all controls were based on the published version of a manuscript. Due to different variables having different degree of missing values, the number of observations used in the regression varies across models.

Manuscript factors. Many manuscript characteristics can be associated with quality or influence the perception of quality. We thus considered the following characteristics:

- The year of submission: Acceptance rates can vary by year. We treated submission year as a categorical variable ("2013” was used as the reference category). For Journal B, for which we lacked data on year of submission, we used year of publication.

- The number of authors: Team size may be related to both the quality and type of submission ${ }^{47 / 64}$. We thus controlled for the number of authors for each submission.

- The title length: number of words in the title of a manuscript.

- The abstract length: number of words in the abstract of a manuscript.

- The abstract readability: We used two measures of abstract readability: (1) the FleschKincaid reading ease score, which estimates the grade-level needed to understand the pas- 
sage, (2) the type-token ratio, which is a measure of lexical variety and complexity.

Last author prestige. Both journals use single-blind reviewing. Many author-level attributes can influence the perception of manuscript quality and the subsequent acceptance decision. For example, studies of the effects of blinding show that high status authors benefit when their identity is visible ${ }^{33}$. We thus controlled for several factors related to author prestige.

- Author rank: this metric is provided by MAG, which estimates the relative importance of an author in the heterogeneous citation network including papers, authors, and affiliations 55 . Note that the author's total number of citations was collinear with the author's rank in a multicollinearity test, thus is excluded in the model.

- Author's previous publications: we approximated author's research experience as the total number of publications by the time of submission (the publication year was used for Journal B). We used all papers indexed in MAG to count authors' prior publications.

- Author's previous publications at Journal A/B: we counted the number of Journal A/B publications up to the submission year (the publication year was used for Journal B). This variable captures the last author's degree of recognition at a specific journal and their familiarity of the journal's style and mission. Based on the so called "Matthew effect" in science ${ }^{65}$, we hypothesized that authors with more prior publications at a journal were more likely to publish there again.

- Author's affiliation rank: similar to the author rank, we also considered the rank of their affiliations provided by MAG. In case of multiple affiliations for the last author, we used the one with the highest rank (a smaller value indicates a higher rank).

- Author's affiliation location: authors from U.S. affiliations were shown to be favored in peer reviews 30 . This geographical bias could potentially explain the disparities (if any) for ethnic minority authors since they were more likely to be affiliated with non-U.S. institutions than 
British-origin authors. We inferred the location (country) of author's institution using the latitude and longitude information provided in MAG, with three categories: (1) Domestic (in the U.S.), (2) International, (3) Unknown. When an author has multiple affiliations, we classified them as "U.S." if there was at least one U.S. affiliation. If the location cannot be inferred for any affiliation due to missing data, we classified them as "Unknown." We used "U.S." as the reference category in the regressions.

Paper topics. Manuscripts in trending areas may be more likely to be accepted than others. We thus controlled for research topics and domains. MAG provides a list of keywords for each paper with associated confidence score between 0 and 1. For each journal, we focused on the most common keywords used by at least 500 published papers (accepted and rejected). There are 13 and 26 keywords for Journal A and Journal B, respectively. Each keyword was used as a variable in the regression, whose value is the keyword's confidence score for the paper.

Paper quality. Quality is one of the most important factors in determining acceptance. Quality is a subjective evaluation criterion that cannot be quantified with a single metric. We therefore approximated paper quality along three dimensions: (1) Novelty, (2) Disruption, (3) Citations.

(1) Novelty. Most journals aim to publish novel research in their stated missions. We adopted the widely used novelty measure proposed by Uzzi et al. ${ }^{46}$, which defines paper novelty as the "atypical combinations" of existing knowledge. This measure has been shown to correlate with subjective perceptions of novelty

This measure considers pairwise combinations of cited journals in the reference of each paper. Journal pairs that have been rarely combined by the literature in a year are considered to be novel in that year. The novelty score of each journal pair in a given year is calculated by comparing its observed co-citation frequency across all published papers in that year to those expected by pure chance. This method then gives each paper a distribution of novelty scores for all journal pairs in 
its reference. It provides two summary statistics for each paper: (1) the median novelty and (2) the tail novelty (10th percentile).

Uzzi et al. used all research articles published over the 1950-2000 period by 15,613 journals indexed in the Thomson Reuters Web of Science (WoS) database ${ }^{46}$. Here, we used the MAG database to quantify the novelty of published papers submitted to Journal A and Journal B. MAG indexes 48,757 journals which cover journal articles, working papers, book chapters, and other article types. The scope of coverage by MAG clearly surpasses the WoS database ${ }^{56}$.

To make the literature size comparable to that of Uzzi et al., we focused on all 21,357 journals in MAG that are also indexed in the Scopus database (matched based on ISSN). We thus limited the analysis to all papers published in $21 \mathrm{~K}$ journals, and only consider, for each paper, its references to those journals in MAG. The literature pool for the novelty calculation consists of 10,144,021 citing papers published in the 2013-2019 period, and their 311,074,438 reference pairs (note that the cited papers were published between 1950 and 2019, which is the same as Uzzi et al.). Our novelty-related factors are:

- Tail novelty: the 10th percentile novelty score of a paper;

- Median novelty: the 50th percentile novelty score of a paper;

- Number of references: this variable is by definition related to the Uzzi novelty measure as it affects the number of journal combinations. We thus controlled for the total number of cited papers in a paper's reference.

- Number of unique journals cited: similarly, we also controlled for the number of unique journals cited by a paper. This variable captures the breadth of existing knowledge that a paper builds on. It also approximates the interdisciplinarity of a paper.

- Number of Journal A/Journal B papers cited: a manuscript that cites many papers from a journal to which it is submitted is probably very relevant to that journal's topic of interest. It 
also reflects whether the editor is familiar with the research topic. We thus controlled for the number of cited papers from the focal journal.

(2) Disruption. While the Uzzi novelty defines innovation as combinations of existing knowledge in the space of literature, this concept does not capture other types of innovation and creativity. Disruption is another notion that has been introduced for the evaluation of novel contributions. The disruption measure was initially designed in the study of patented inventions ${ }^{67}$, and has been recently introduced in the analysis of scientific papers ${ }^{47}$. It measures innovation across time by assessing the degree to which a paper destabilizes existing knowledge it builds upon. A paper is considered disruptive if it introduces something new that shadows the attention (citations) to its cited papers ("parents") after publication. It has been validated through evaluation tasks, such as consulting domain experts and examining Nobel-prize-winning papers.

Specifically, for a given paper and its cited papers (references), the measure examines three groups of papers $\left(G_{i}, G_{j}, G_{k}\right)$ published after the focal paper. Papers in $G_{i}$ only cited the focal paper; papers in $G_{j}$ cited both the focal paper and its references; papers in $G_{k}$ only cited the focal paper's references. The disruption is calculated as: $\frac{\left|G_{i}\right|-\left|G_{j}\right|}{\left|G_{i}\right|+\left|G_{j}\right|+\left|G_{k}\right|}$, where $\left|G_{n}\right|$ is the number of papers in group $G_{n}$.

(3) Citations. Innovation alone cannot capture many different aspects of scholarly contributions. Another important dimension of paper quality is its scientific impact, which is often measured as the observed citation count - the number of times that the research community has credited a paper for its inspiration of new ideas. High impact papers are often considered to be of high quality. We thus considered the total number of citations up to June 2019 (the MAG access date) in log scale for each published paper. The results presented in the main paper are similar when using the raw number of citations. Since citations are heavily influenced by the time of publication, we also controlled for the publication year of a paper (treated as a categorical variable with "2013" used as the reference category). 


\section{References}

1. Valantine, H. A. \& Collins, F. S. National institutes of health addresses the science of diversity. PNAS 112, 12240-12242 (2015).

2. Boekhout, H., van der Weijden, I. \& Waltman, L. Gender differences in scientific careers: A large-scale bibliometric analysis (2021). 2106.12624.

3. Ley, T. J. \& Hamilton, B. H. The gender gap in nih grant applications. Science 322 (2008).

4. Shen, H. Inequality quantified: Mind the gender gap. Nature News 495, 22 (2013).

5. Huang, J., Gates, A. J., Sinatra, R. \& Barabási, A.-L. Historical comparison of gender inequality in scientific careers across countries and disciplines. PNAS 117, 4609-4616 (2020).

6. Xie, Y. "undemocracy": inequalities in science. Science 344, 809-810 (2014).

7. Ginther, D. K. et al. Publications as predictors of racial and ethnic differences in nih research awards. PLoS One 13, e0205929 (2018).

8. Willis, M. Gender and racial/ethnic disparities in rates of publishing and inclusion in scientific review processes. Translational Issues in Psychological Science (2021).

9. Merton, R. K. On social structure and science (University of Chicago Press, 1996).

10. Lee, C. J., Sugimoto, C. R., Zhang, G. \& Cronin, B. Bias in peer review. Journal of the American Society for Information Science and Technology 64, 2-17 (2013).

11. Moss-Racusin, C. A., Dovidio, J. F., Brescoll, V. L., Graham, M. J. \& Handelsman, J. Science faculty's subtle gender biases favor male students. PNAS 109, 16474-16479 (2012).

12. Ginther, D. K. et al. Race, ethnicity, and nih research awards. Science 333 (2011).

13. Oliveira, D. F., Ma, Y., Woodruff, T. K. \& Uzzi, B. Comparison of national institutes of health grant amounts to first-time male and female principal investigators. JAMA 321, 898-900 (2019). 
14. Jones, B. F. The burden of knowledge and the "death of the renaissance man": Is innovation getting harder? The Review of Economic Studies 76, 283-317 (2009).

15. Wuchty, S., Jones, B. F. \& Uzzi, B. The increasing dominance of teams in production of knowledge. Science 316, 1036-1039 (2007).

16. Jones, B. F., Wuchty, S. \& Uzzi, B. Multi-university research teams: Shifting impact, geography, and stratification in science. Science 322, 1259-1262 (2008).

17. Tversky, A. \& Kahneman, D. Judgment under uncertainty: Heuristics and biases. Science 185, 1124-1131 (1974).

18. Gigerenzer, G. \& Todd, P. M. Fast and frugal heuristics: The adaptive toolbox. In Simple heuristics that make us smart, 3-34 (Oxford University Press, 1999).

19. Bingham, C. B. \& Eisenhardt, K. M. Rational heuristics: the 'simple rules' that strategists learn from process experience. Strategic Management Journal 32, 1437-1464 (2011).

20. Knobloch-Westerwick, S., Glynn, C. J. \& Huge, M. The matilda effect in science communication: an experiment on gender bias in publication quality perceptions and collaboration interest. Science Communication 35, 603-625 (2013).

21. Nielsen, M. W. et al. Opinion: Gender diversity leads to better science. PNAS 114, 1740-1742 (2017).

22. Hoppe, T. A. et al. Topic choice contributes to the lower rate of nih awards to africanamerican/black scientists. Science Advances 5, eaaw7238 (2019).

23. Koning, R., Samila, S. \& Ferguson, J.-P. Who do we invent for? patents by women focus more on women's health, but few women get to invent. Science 372, 1345-1348 (2021).

24. Correll, S. J. \& Ridgeway, C. L. Expectation states theory. In Handbook of social psychology, 29-51 (Springer, 2006). 
25. Tilcsik, A. Statistical discrimination and the rationalization of stereotypes. American Sociological Review 0003122420969399 (2020).

26. Foschi, M. Double standards for competence: Theory and research. Annual Review of Sociology 26, 21-42 (2000).

27. Card, D., DellaVigna, S., Funk, P. \& Iriberri, N. Are referees and editors in economics gender neutral? The Quarterly Journal of Economics 135, 269-327 (2020).

28. Helmer, M., Schottdorf, M., Neef, A. \& Battaglia, D. Gender bias in scholarly peer review. Elife 6, e21718 (2017).

29. Day, A., Corbett, P. \& Boyle, J. Is there a gender gap in chemical sciences scholarly communication? Chemical Science 11, 2277-2301 (2020).

30. Link, A. M. Us and non-us submissions: an analysis of reviewer bias. JAMA 280, 246-247 (1998).

31. Ross, J. S. et al. Effect of blinded peer review on abstract acceptance. JAMA 295, 1675-1680 (2006).

32. Okike, K., Hug, K. T., Kocher, M. S. \& Leopold, S. S. Single-blind vs double-blind peer review in the setting of author prestige. JAMA 316, 1315-1316 (2016).

33. Tomkins, A., Zhang, M. \& Heavlin, W. D. Reviewer bias in single-versus double-blind peer review. PNAS 114, 12708-12713 (2017).

34. Teplitskiy, M., Acuna, D., Elamrani-Raoult, A., Körding, K. \& Evans, J. The sociology of scientific validity: How professional networks shape judgement in peer review. Research Policy 47, 1825-1841 (2018).

35. Hofstra, B. et al. The diversity-innovation paradox in science. Proceedings of the National Academy of Sciences 117, 9284-9291 (2020). 
36. Witteman, H. O., Hendricks, M., Straus, S. \& Tannenbaum, C. Are gender gaps due to evaluations of the applicant or the science? a natural experiment at a national funding agency. The Lancet 393, 531-540 (2019).

37. Williams, W. M. \& Ceci, S. J. National hiring experiments reveal 2: 1 faculty preference for women on stem tenure track. PNAS 112, 5360-5365 (2015).

38. Forscher, P. S., Cox, W. T., Brauer, M. \& Devine, P. G. Little race or gender bias in an experiment of initial review of nih r01 grant proposals. Nature Human Behaviour 3, 257-264 (2019).

39. Witze, A. Research gets increasingly international. Nature News (2016).

40. Tollefson, J. China declared world's largest producer of scientific articles. Nature $\mathbf{5 5 3}$ (2018).

41. Elsevier. URL https://www.elsevier.com/editors-update/story/ peer-review/what-researchers-think-about-the-peer-review-process.

42. Torvik, V. I. \& Agarwal, S. Ethnea-an instance-based ethnicity classifier based on geo-coded author names in a large-scale bibliographic database. International Symposium on Science of Science (2016).

43. Wu, K. J. Scientific journals commit to diversity but lack the data (2020). URL https:// WwW.nytimes.com/2020/10/30/science/diversity-science-journals. html.

44. Wren, J. D. et al. The write position: A survey of perceived contributions to papers based on byline position and number of authors. EMBO Reports 8, 988-991 (2007).

45. Sekara, V. et al. The chaperone effect in scientific publishing. PNAS 115, 12603-12607 (2018).

46. Uzzi, B., Mukherjee, S., Stringer, M. \& Jones, B. Atypical combinations and scientific impact. Science 342, 468-472 (2013). 
47. Wu, L., Wang, D. \& Evans, J. A. Large teams develop and small teams disrupt science and technology. Nature 566, 378 (2019).

48. Harris, M., Macinko, J., Jimenez, G., Mahfoud, M. \& Anderson, C. Does a research article's country of origin affect perception of its quality and relevance? a national trial of us public health researchers. BMJ Open 5, e008993 (2015).

49. Harris, M. et al. Explicit bias toward high-income-country research: a randomized, blinded, crossover experiment of english clinicians. Health Affairs 36, 1997-2004 (2017).

50. Hengel, E. \& Moon, E. Gender and quality at top economic journals. Tech. Rep. (2019).

51. Larivière, V. \& Gingras, Y. The impact factor's matthew effect: A natural experiment in bibliometrics. Journal of the American Society for Information Science and Technology 61, 424-427 (2010).

52. Teplitskiy, M., Peng, H., Blasco, A. \& Lakhani, K. Does the scientific community resist change? testing the anti-novelty hypothesis in peer review. Working Paper (2021).

53. Bodenhausen, G. V. \& Wyer, R. S. Effects of stereotypes in decision making and informationprocessing strategies. Journal of Personality and Social Psychology 48, 267 (1985).

54. Bohnet, I., Van Geen, A. \& Bazerman, M. When performance trumps gender bias: Joint vs. separate evaluation. Management Science 62, 1225-1234 (2016).

55. Wang, K. et al. A review of microsoft academic services for science of science studies. Frontiers in Big Data 2, 45 (2019).

56. Visser, M., van Eck, N. J. \& Waltman, L. Large-scale comparison of bibliographic data sources: Scopus, web of science, dimensions, crossref, and microsoft academic. arXiv:2005.10732 (2020).

57. Goodman, S. N., Berlin, J., Fletcher, S. W. \& Fletcher, R. H. Manuscript quality before and after peer review and editing at annals of internal medicine. Annals of Internal Medicine 121, 11-21 (1994). 
58. Ellison, G. Evolving standards for academic publishing: A q-r theory. Journal of Political Economy 110, 994-1034 (2002).

59. Siler, K., Lee, K. \& Bero, L. Measuring the effectiveness of scientific gatekeeping. Proceedings of the National Academy of Sciences 112, 360-365 (2015).

60. Strang, D. \& Siler, K. Revising as reframing: original submissions versus published papers in administrative science quarterly, 2005 to 2009. Sociological Theory 33, 71-96 (2015).

61. Teplitskiy, M. Frame search and re-search: How quantitative sociological articles change during peer review. The American Sociologist 47, 264-288 (2016).

62. Ambekar, A., Ward, C., Mohammed, J., Male, S. \& Skiena, S. Name-ethnicity classification from open sources. In $K D D, 49-58$ (2009).

63. Treeratpituk, P. \& Giles, C. L. Name-ethnicity classification and ethnicity-sensitive name matching. In Twenty-Sixth AAAI Conference on Artificial Intelligence (2012).

64. AlShebli, B. K., Rahwan, T. \& Woon, W. L. The preeminence of ethnic diversity in scientific collaboration. Nature Communications 9, 5163 (2018).

65. Merton, R. K. The matthew effect in science: The reward and communication systems of science are considered. Science 159, 56-63 (1968).

66. Bornmann, L., Tekles, A., Zhang, H. H. \& Fred, Y. Y. Do we measure novelty when we analyze unusual combinations of cited references? a validation study of bibliometric novelty indicators based on f1000prime data. Journal of Informetrics 13, 100979 (2019).

67. Funk, R. J. \& Owen-Smith, J. A dynamic network measure of technological change. Management Science 63, 791-817 (2017).

Acknowledgements The authors thank David Jurgens and Daniel M. Romero for helpful discussion and suggestions. We also thank the two journals for providing their peer review data. This study was approved by the University of Michigan institutional review board. 
Author Contributions H.P. and M.T. conceived the idea and designed the study; H.P. performed the analyses; H.P., M.T., and K.L. wrote the manuscript.

Competing Interests The authors declare no competing interests.

Additional Information Supplemental material is available for this paper.

Materials \& Correspondence Correspondence and requests for materials should be addressed to M.T., tepl@umich.edu 


\section{Acceptance in Top Journals Shows Large Disparities across Name-inferred Ethnicities (Supplemental Material)}

Hao Peng, Karim Lakhani, Misha Teplitskiy

(Dated: July 2, 2021)

\section{SI TABLES}

\begin{tabular}{|l|l|}
\hline Broad Ethnic Category & Individual Ethnicity \\
\hline African & African \\
British-origin & English \\
Chinese & Chinese \\
non-Chinese East Asian & Indonesian, Japanese, Korean, Mongolian, Thai, Vietnamese \\
Western \& Northern European & German, French, Dutch, Nordic, Baltic \\
Southern European & Greek, Hispanic, Italian \\
Eastern European & Romanian, Hungarian, Slav \\
Indian & Indian \\
Middle Eastern & Arab, Israeli, Turkish \\
Caribbean & Caribbean \\
Polynesian & Polynesian \\
Unknown & Note: names are unrecognized by Ethnea. \\
\hline
\end{tabular}

TABLE S1. 26 individual ethnicities were grouped into the 11 broad ethnic categories. Two groups, Caribbean and Polynesian, were excluded due to less than 5 observations. 


\begin{tabular}{|c|c|c|c|c|c|}
\hline & Model 1 & Model 2 & Model 3 & Model 4 & Model 5 \\
\hline African & -0.547 & -0.542 & 0.320 & 0.265 & 0.310 \\
\hline Chinese & $-1.109^{* * *}$ & $-1.057^{* * *}$ & $-0.351^{* *}$ & $-0.374^{* *}$ & $-0.390^{* *}$ \\
\hline non-Chinese East Asian & $-1.758^{* * *}$ & $-1.701^{* * *}$ & $-0.922^{* * *}$ & $-0.863^{* * *}$ & $-0.686^{* * *}$ \\
\hline Eastern European & $-0.656^{* * *}$ & $-0.710^{* * *}$ & -0.219 & -0.232 & -0.231 \\
\hline Indian & $-0.457^{* * *}$ & $-0.437^{* * *}$ & 0.004 & -0.025 & 0.050 \\
\hline Middle Eastern & $-0.273^{* *}$ & $-0.218^{*}$ & $0.466^{* * *}$ & $0.414^{* *}$ & $0.392^{*}$ \\
\hline Southern European & $-0.467^{* * *}$ & $-0.468^{* * *}$ & -0.024 & 0.006 & -0.127 \\
\hline Western \& Northern European & $-0.351^{* * *}$ & $-0.365^{* * *}$ & -0.017 & 0.002 & -0.058 \\
\hline Unknown Ethnicity & 0.124 & 0.029 & 0.122 & 0.307 & -0.383 \\
\hline Female & $-0.131^{*}$ & -0.119 & 0.038 & 0.040 & 0.051 \\
\hline Unknown Gender & $-0.166^{*}$ & $-0.171^{*}$ & -0.164 & -0.157 & -0.251 \\
\hline Submission year 2014 & & -0.050 & $-1.273^{* * *}$ & $-1.292^{* * *}$ & -0.300 \\
\hline Submission year 2015 & & $-0.262^{* * *}$ & $-1.459^{* * *}$ & $-1.401^{* * *}$ & 0.280 \\
\hline Submission year 2016 & & $-0.398^{* * *}$ & $-1.584^{* * *}$ & $-1.485^{* * *}$ & $1.262^{* * *}$ \\
\hline Submission year 2017 & & $-0.233^{* *}$ & $-1.296^{* * *}$ & $-1.079^{* * *}$ & $2.557^{* * *}$ \\
\hline Submission year 2018 & & $-0.806^{* * *}$ & $-0.855^{* * *}$ & $-0.581^{* * *}$ & $4.015^{* * *}$ \\
\hline Number of authors & & $0.012^{* * *}$ & $0.012^{* *}$ & $0.010^{*}$ & $-0.020^{* * *}$ \\
\hline Title length & & $-0.131^{* * *}$ & $-0.140^{* * *}$ & $-0.146^{* * *}$ & $-0.115^{* * *}$ \\
\hline Abstract length & & $-0.018^{* * *}$ & $-0.025^{* * *}$ & $-0.030^{* * *}$ & $-0.034^{* * *}$ \\
\hline Flesch-Kincaid score & & $-0.006^{* * *}$ & $-0.004^{* *}$ & $-0.008^{* * *}$ & $-0.008^{* * *}$ \\
\hline Type-Token ratio & & $3.522^{* * *}$ & $4.554^{* * *}$ & $3.686^{* * *}$ & $2.960^{* * *}$ \\
\hline Last author rank & & & $-0.0002^{* * *}$ & $-0.0002^{* * *}$ & 0.00000 \\
\hline Last author affiliation rank & & & -0.00003 & -0.00002 & 0.00000 \\
\hline Last author affiliation intl. (location) & & & $-0.406^{* * *}$ & $-0.466^{* * *}$ & $-0.444^{* * *}$ \\
\hline Last author affiliation unknown (location) & & & 0.715 & 0.288 & -0.086 \\
\hline Last author prior publications. & & & $-0.002^{* * *}$ & $-0.001^{* * *}$ & -0.0005 \\
\hline Last author prior Journal A publications & & & $0.042^{* * *}$ & $0.041^{* * *}$ & $0.031^{* * *}$ \\
\hline Tail novelty & & & & & $-0.005^{* * *}$ \\
\hline Median novelty & & & & & -0.001 \\
\hline Disruption & & & & & 0.118 \\
\hline Number of references & & & & & $0.018^{* * *}$ \\
\hline Number of unique J. cited & & & & & $-0.042^{* * *}$ \\
\hline Number of J. A papers cited & & & & & $0.043^{* *}$ \\
\hline Total citations $(\log )$ & & & & & $1.124^{* * *}$ \\
\hline Publication year 2014 & & & & & $-0.520^{*}$ \\
\hline Publication year 2015 & & & & & $-0.987^{* * *}$ \\
\hline Publication year 2016 & & & & & $-1.211^{* * *}$ \\
\hline Publication year 2017 & & & & & $-1.818^{* * *}$ \\
\hline Publication year 2018 & & & & & $-1.132^{* *}$ \\
\hline Publication year 2019 & & & & & 0.056 \\
\hline Intercept & $-1.274^{* * *}$ & -0.164 & $5.680^{* * *}$ & $4.923^{* * *}$ & $-2.938^{*}$ \\
\hline Keywords included & $\mathrm{N}$ & $\mathrm{N}$ & $\mathrm{N}$ & $\mathrm{Y}$ & $\mathrm{Y}$ \\
\hline Observations & 16,956 & 16,954 & 7,062 & 7,062 & 6,947 \\
\hline
\end{tabular}

TABLE S2. Coefficients of five increasing-complexity regression models in predicting if a manuscript was finally accepted by Journal $A$. Coefficients for 13 keywords are omitted to ensure journal anonymity. Significance levels: $* * *$ $\mathrm{p}<0.001, * * \mathrm{p}<0.01$, and $* \mathrm{p}<0.05$. 


\begin{tabular}{|c|c|c|c|c|c|}
\hline & Model 1 & Model 2 & Model 3 & Model 4 & Model 5 \\
\hline African & -0.418 & -0.185 & -0.051 & -0.069 & 0.216 \\
\hline Chinese & $-1.025^{* * *}$ & $-1.100^{* * *}$ & $-0.887^{* * *}$ & $-0.970^{* * *}$ & $-0.960^{* * *}$ \\
\hline non-Chinese East Asian & $-1.107^{* * *}$ & $-1.136^{* * *}$ & $-0.743^{* * *}$ & $-0.724^{* * *}$ & $-0.612^{* * *}$ \\
\hline Eastern European & $-0.470^{* * *}$ & $-0.599^{* * *}$ & $-0.526^{* * *}$ & $-0.653^{* * *}$ & $-0.691^{* * *}$ \\
\hline Indian & $-0.730^{* * *}$ & $-0.761^{* * *}$ & $-0.522^{* * *}$ & $-0.449^{* *}$ & $-0.423^{*}$ \\
\hline Middle Eastern & $-0.399^{* * *}$ & $-0.448^{* * *}$ & -0.214 & -0.254 & -0.146 \\
\hline Southern European & $-0.533^{* * *}$ & $-0.636^{* * *}$ & $-0.330^{* * *}$ & -0.213 & $-0.246^{*}$ \\
\hline Western \& Northern European & $-0.288^{* * *}$ & $-0.333^{* * *}$ & -0.133 & -0.089 & -0.052 \\
\hline Ethnicity Unknown & $-2.009^{* * *}$ & $-1.545^{* * *}$ & -0.616 & -0.619 & 0.118 \\
\hline Female & -0.076 & -0.052 & 0.002 & 0.090 & 0.144 \\
\hline Gender Unknown & $-0.527^{* * *}$ & $-0.486^{* * *}$ & $-0.326^{* * *}$ & $-0.228^{*}$ & -0.149 \\
\hline Number of authors & & $0.026^{* * *}$ & $0.023^{* * *}$ & $0.035^{* * *}$ & $0.034^{* * *}$ \\
\hline Title length & & $-0.056^{* * *}$ & $-0.038^{* * *}$ & $-0.040^{* * *}$ & $-0.038^{* * *}$ \\
\hline Abstract length & & $-0.025^{* * *}$ & $-0.027^{* * *}$ & $-0.030^{* * *}$ & $-0.029^{* * *}$ \\
\hline Flesch-Kincaid score & & $-0.007^{* * *}$ & $-0.006^{* * *}$ & $-0.009^{* * *}$ & $-0.011^{* * *}$ \\
\hline Type-Token ratio & & $-7.513^{* * *}$ & $-4.420^{* * *}$ & $-4.244^{* * *}$ & $-3.346^{* * *}$ \\
\hline Last author rank & & & $-0.0002^{* * *}$ & $-0.0001^{* * *}$ & $-0.0001^{* *}$ \\
\hline Last author affiliation rank & & & $-0.0001^{* * *}$ & $-0.0001^{* * *}$ & $-0.0001^{* *}$ \\
\hline Last author affiliation intl. (location) & & & $-0.448^{* * *}$ & $-0.441^{* * *}$ & $-0.459^{* * *}$ \\
\hline Last author affiliation unknown (location) & & & 0.342 & -0.044 & -0.184 \\
\hline Last author prior publications. & & & $-0.002^{* * *}$ & $-0.001^{* * *}$ & $-0.001^{*}$ \\
\hline Last author prior Journal B publications & & & $0.278^{* * *}$ & $0.252^{* * *}$ & $0.198^{* * *}$ \\
\hline Tail novelty & & & & & -0.001 \\
\hline Median novelty & & & & & $0.002^{* * *}$ \\
\hline Disruption & & & & & -0.168 \\
\hline Number of references & & & & & $-0.011^{*}$ \\
\hline Number of unique J. cited & & & & & -0.014 \\
\hline Number of J. B papers cited & & & & & $0.089^{*}$ \\
\hline Total citations $(\log )$ & & & & & $0.464^{* * *}$ \\
\hline Publication year 2014 & & & & & $-1.191^{* * *}$ \\
\hline Publication year 2015 & & & & & $-1.120^{* * *}$ \\
\hline Publication year 2016 & & & & & $-0.536^{* *}$ \\
\hline Publication year 2017 & & & & & $-0.368^{*}$ \\
\hline Publication year 2018 & & & & & $0.510^{*}$ \\
\hline Publication year 2019 & & & & & $1.437^{* * *}$ \\
\hline Intercept & $0.347^{* * *}$ & $9.193^{* * *}$ & $10.628^{* * *}$ & $7.788^{* * *}$ & $5.242^{* * *}$ \\
\hline Keywords included & $\mathrm{N}$ & $\mathrm{N}$ & $\mathrm{N}$ & $\mathrm{Y}$ & $\mathrm{Y}$ \\
\hline Observations & 14,269 & 8,874 & 7,195 & 7,195 & 6,365 \\
\hline
\end{tabular}

TABLE S3. Coefficients of five increasing-complexity regression models in predicting if a manuscript was finally accepted by Journal B. Coefficients for 26 keywords are omitted to ensure journal anonymity. Significance levels: $* * *$ $\mathrm{p}<0.001,{ }^{* *} \mathrm{p}<0.01$, and $* \mathrm{p}<0.05$.

\begin{tabular}{|l|c|c|r|}
\hline Ethnicity & U.S.-based & International & p-value \\
\hline Southern European & $-0.35^{*}$ & 0.26 & 0.035 \\
Western \& Northern European & -0.15 & 0.08 & 0.306 \\
Eastern European & -0.28 & -0.08 & 0.660 \\
non-Chinese East Asian & $-0.82^{*}$ & -0.52 & 0.485 \\
Chinese & $-0.47^{* *}$ & -0.13 & 0.334 \\
Middle Eastern & $0.62^{* *}$ & 0.24 & 0.303 \\
Indian & -0.04 & 0.44 & 0.314 \\
African & -0.59 & 1.12 & 0.361 \\
\hline
\end{tabular}

TABLE S4. The ethnicity coefficients of Model 5 in predicting the final acceptance at Journal A. A separate model is trained for the submissions from U.S.-based authors (4,075 observations), and the international authors subset (2,942 observations), respectively. Stars indicate the significance level for each coefficient $(* * * \mathrm{p}<0.001, * * \mathrm{p}<0.01$, and $*$ $\mathrm{p}<0.05)$. The $\mathrm{p}$-values are based on the statistical test of differences in coefficients between two models [1]. 


\begin{tabular}{|l|c|c|r|}
\hline Ethnicity & U.S.-based & International & p-value \\
\hline Southern European & -0.24 & $-0.34^{*}$ & 0.719 \\
Western \& Northern European & -0.02 & -0.16 & 0.511 \\
Eastern European & $-0.68^{* *}$ & $-0.74^{*}$ & 0.864 \\
non-Chinese East Asian & $-0.76^{* *}$ & $-0.70^{* * *}$ & 0.855 \\
Chinese & $-0.91^{* * *}$ & $-1.19^{* * *}$ & 0.294 \\
Middle Eastern & -0.32 & -0.05 & 0.452 \\
Indian & -0.38 & $-0.65^{*}$ & 0.481 \\
African & -1.44 & 1.31 & 0.116 \\
\hline
\end{tabular}

TABLE S5. The ethnicity coefficients of Model 5 in predicting the final acceptance at Journal B. A separate model is trained for the submissions from U.S.-based authors (2,812 observations), and the international authors subset (3,549 observations), respectively. Stars indicate the significance level for each coefficient $\left(* * * \mathrm{p}<0.001,{ }^{* *} \mathrm{p}<0.01\right.$, and $*$ $\mathrm{p}<0.05)$. The $\mathrm{p}$-values are based on the statistical test of differences in coefficients between two models [1]. 
[1] Clifford C Clogg, Eva Petkova, and Adamantios Haritou. Statistical methods for comparing regression coefficients between models. American Journal of Sociology, 100(5):1261-1293, 1995. 